\title{
Searches for Squarks and Gluinos at the Tevatron
}

\author{
Eric Kajfasz* \\ CPPM (Centre de Physique des Particules de Marseille, France.) \\ On behalf of the $C D F$ and $D 0$ collaborations ${ }^{\dagger}$ \\ E-mail: kajfasz@cppm.in2p3.fr
}

\begin{abstract}
We show results on searches for squarks and gluinos performed on up to $2.1 \mathrm{fb}^{-1}$ of data from proton-antiproton collisions at $1.96 \mathrm{TeV}$ center of mass energy, collected using the CDF and D0 detectors at the Fermilab Tevatron Run II. Events with multiple jets of hadrons and large missing transverse energy in the final state are studied within the framework of minimal supergravity (mSUGRA) and assuming R-parity conservation. At D0, the search for squarks has also been performed in the topology of multijet events accompanied by large missing transverse energy and at least one tau lepton decaying hadronically. At CDF, a generic search utilizing exclusive dijets events with large missing transverse energy has also been studied within the Minimal Supersymmetric Standard Model (MSSM). Published and preliminary results are presented.
\end{abstract}

The 2009 Europhysics Conference on High Energy Physics,

July 16 - 222009

Krakow, Poland

\footnotetext{
* Speaker.

${ }^{\dagger}$ Many thanks to the D0 New Phenomena and CDF Exotics groups and to their conveners for their help in preparing the talk.
} 


\section{Introduction}

Supersymmetry (SUSY) [1] is an extension of the Standard Model that predicts the existence of still undiscovered particles, including scalar quarks (squarks, $\tilde{q}$ ) and fermionic gluons (gluinos, $\tilde{g}$ ). Since colored, if sufficiently light, $\tilde{q}$ and $\tilde{g}$ can be copiously produced at hadron colliders. If R-parity is conserved, the lightest supersymmetric particle (LSP), here assumed to be the lightest neutralino $\left(\tilde{\chi}_{1}^{0}\right)$, is stable. Generally, $\tilde{q}$ and $\tilde{g}$ cascade decay in jets and the LSP, giving rise to events with jets and a large missing transverse energy (MET), as studied in sections 2 and 4. But in some regions of the SUSY parameter space, $\tilde{q}$ and $\tilde{g}$ cascade decays can also lead to final states with leptons (see section 3).

In the Minimal Supersymmetric Standard Model (MSSM), many new parameters are introduced. However in the minimal supergravity (mSUGRA) framework, where supersymmetry breaking is achieved via gravitational interactions, only five parameters determine the low energy phenomenology, namely: $m_{0}$, the common scalar mass, $m_{1 / 2}$, the common gaugino mass, and $A_{0}$, the common trilinear coupling at the Grand Unification (GUT) scale, $\tan \beta$, the ratio of the Higgs vacuum expectation values, and $\operatorname{sign}(\mu)$, the sign of the higgsino mass term.

\section{Jets + MET analyses}

The inclusive search of $\tilde{q}$ and $\tilde{g}$ in the jets + MET channel has been performed by CDF and D0 with data samples of about $2 \mathrm{fb}^{-1}$. In each case, one studies separately MET $+\geq 2$ jets, MET $+\geq 3$ jets, MET $+\geq 4$ jets events. Non collision background is removed, MET is required not to be aligned with jets (QCD background reduction) and leptons are vetoed (EW and top background reduction). Cuts on jets transverse energy (ET), on HT (scalar sum of jets ET) and MET are optimized, the values of which are given in table 1.

\begin{tabular}{|c||c|c|c||c|c|c|}
\hline \multicolumn{1}{|c||}{} & \multicolumn{3}{c||}{ CDF } & \multicolumn{3}{c|}{ D0 } \\
\hline Analysis & $\begin{array}{c}\text { HT } \\
(\mathrm{GeV})\end{array}$ & $\begin{array}{c}\text { MET } \\
(\mathrm{GeV})\end{array}$ & $\begin{array}{c}\text { Jets ET } \\
(\mathrm{GeV})\end{array}$ & $\begin{array}{c}\text { HT } \\
(\mathrm{GeV})\end{array}$ & $\begin{array}{c}\text { MET } \\
(\mathrm{GeV})\end{array}$ & $\begin{array}{c}\text { Jets ET } \\
(\mathrm{GeV})\end{array}$ \\
\hline 2-jets & 330 & 180 & 165,100 & 325 & 225 & 35,35 \\
\hline 3-jets & 330 & 120 & $140,100,25$ & 375 & 175 & $35,35,35$ \\
\hline 4-jets & 280 & 90 & $95,55,55,25$ & 400 & 100 & $35,35,35,20$ \\
\hline
\end{tabular}

Table 1: Optimized cuts on jets ET, on HT (scalar sum of jets ET) and MET in the CDF and DO analyses

After all cuts, the remaining observed events and expected from backgrounds are given in table 2 for the 3 analyses.

\begin{tabular}{|c||c|c||c|c|}
\hline \multicolumn{1}{|c||}{} & \multicolumn{2}{c||}{ CDF $\left(2 \mathrm{fb}^{-1}\right)$} & \multicolumn{2}{c|}{ D0 $\left(2.1 \mathrm{fb}^{-1}\right)$} \\
\hline Analysis & \# Expected & \# Observed & \# Expected & \# Observed \\
\hline 2-jets & $16 \pm 5$ & 18 & $11 \pm 1_{-2}^{+3}$ & 11 \\
\hline 3-jets & $37 \pm 12$ & 38 & $11 \pm 1_{-2}^{+3}$ & 9 \\
\hline 4-jets & $48 \pm 17$ & 45 & $18 \pm 1_{-3}^{+6}$ & 20 \\
\hline
\end{tabular}

Table 2: Expected and Observed number of events in the CDF and DO analyses after optimized cuts applied 
The main source of systematics is the jet energy scale: 10-15 (6-11)\% for background (signal). Since no excess is seen in data over expected background, limits are computed using the results of the three analyses. For each $\tilde{q}-\tilde{g}$ mass, CDF considers the selection which gives the best expected limit; D0 splits the 3 analyses in 7 independant (exclusive) selections and combines them.

In Fig. 1 the left plot shows the D0 limit in the $\mathrm{M}_{\tilde{g}}-\mathrm{M}_{\tilde{q}}$ plane. The yellow band corresponds to the PDF, and to the renormalization and factorization scale uncertainties on the signal NLO crosssection $(25-75 \%)$. In the right plot the result is expressed in the $m_{0}-m_{1 / 2}$ mSUGRA parameter space, showing an improvement w.r.t. LEP measurements for $m_{0}=700-300 \mathrm{GeV}$ and $m_{1 / 2}=$ $125-165 \mathrm{GeV}$. The analysis is published in PLB [2].
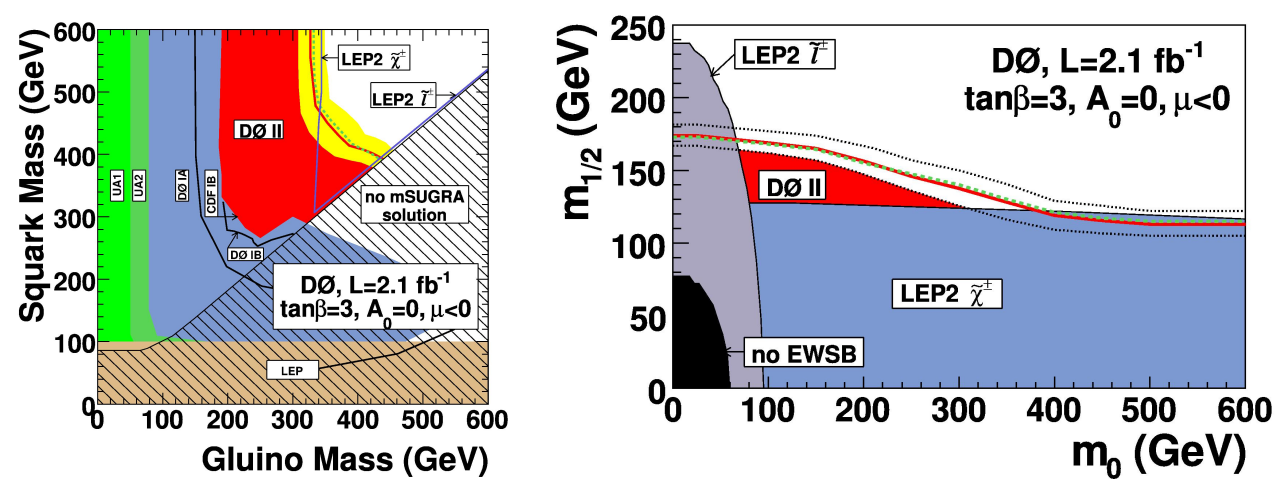

Figure 1: D0 limits on $\tilde{q}$ and $\tilde{g}$ production expressed in the $\mathbf{M}_{\tilde{g}}-\mathbf{M}_{\tilde{q}}$ (left) and $m_{0}-m_{1 / 2}$ (right) planes.

Fig. 2 shows somewhat similar results for the CDF analysis published in PRL[3]: $\mathrm{M}_{\tilde{g}}<280 \mathrm{GeV}(\mathrm{CDF}),<308 \mathrm{GeV}$ (D0), for all $\mathrm{M}_{\tilde{q}}$ and $\mathrm{M}_{\tilde{q}}<380 \mathrm{GeV}(\mathrm{CDF}, \mathrm{D} 0)$, for all $\mathrm{M}_{\tilde{g}}$
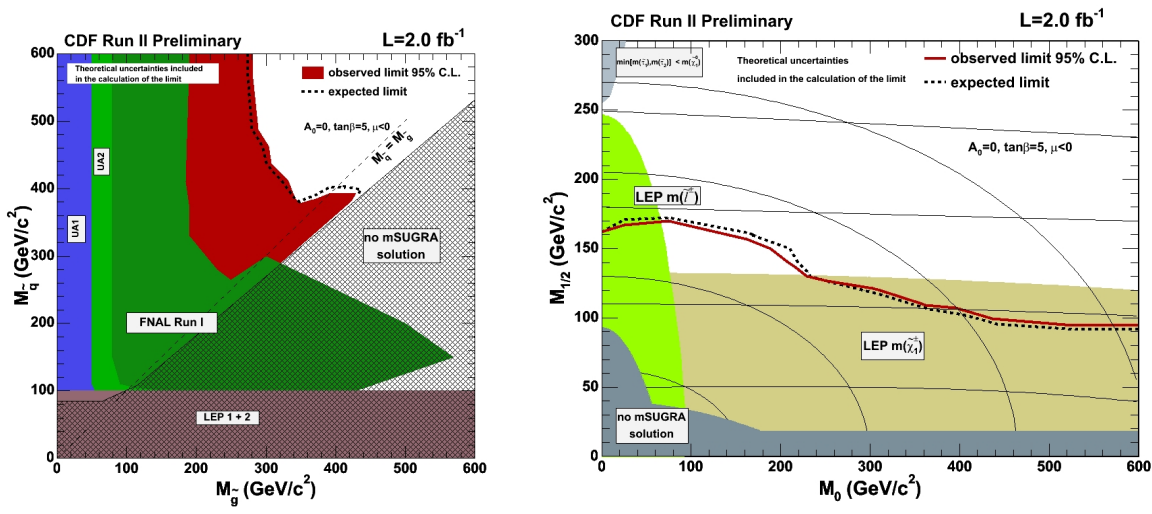

Figure 2: CDF limits on $\tilde{q}$ and $\tilde{g}$ production expressed in the $\mathbf{M}_{\tilde{g}}-\mathbf{M}_{\tilde{q}}$ (left) and $m_{0}-m_{1 / 2}$ (right) planes.

\section{Jets $+\operatorname{tau}(s)+$ MET analysis}

The "tau corridor" is a region of the SUSY parameter space where a large mixing in the tau 
sector results in the lightest stau $\tilde{\tau}_{1}$ to be the next to the lightest SUSY particle (NLSP) and thus makes it possible for it to be produced in the cascade decays of squarks and gluinos, which gives rise to final states with taus in addition to jets and MET. For the D0 "tau" analysis, the event selection requires $\geq 2$ jets and $\geq 1$ hadronically decaying tau(s) in a $0.96 \mathrm{fb}^{-1}$ data sample. The optimization is performed on MET $>175 \mathrm{GeV}$ and ST (sum of the transverse momenta of the 2 leading jets and tau) $>325 \mathrm{GeV}$, ending up in counting 3 data events, in agreement with the $2.3 \pm 0.4$ (stat.) \pm 0.7 (syst.) expected from background. This allows to set limits in the $m_{0}-m_{1 / 2}$ plane (see left plot of Fig. 3) which can be interpreted as a $340 \mathrm{GeV}$ lower limit to the squark mass.
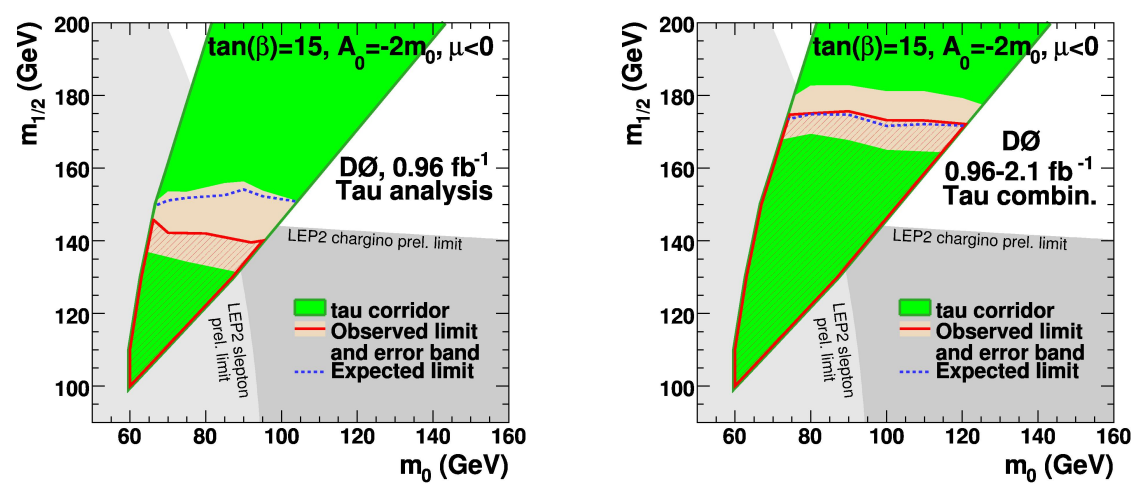

Figure 3: In the $m_{0}-m_{1 / 2}$ plane, expected and observed limits in the "tau corridor" for the "tau" analysis alone (left) and combined with the jets+MET analysis (right)

In order to increase the sensitivity in the tau corridor, since hadronically decaying taus are also detected as jets, this analysis is combined to the D0 $2.1 \mathrm{fb}^{-1}$ jets + MET analysis described in section 2. This results (see right plot of Fig. 3) in limits exceeding the LEP ones and the highest excluded squark mass being $410 \mathrm{GeV}$. This D0 analysis has been accepted for publication in PLB [4].

\section{Exclusive dijet + MET analysis}

CDF performs a generic search for new physics based on $2.0 \mathrm{fb}^{-1}$ of data studying events with an exclusive dijet + MET signature. A low kinematic region event sample is selected using $\mathrm{HT}>125 \mathrm{GeV}$ and MET $>80 \mathrm{GeV}$. A separate search is also performed in a high kinematic region defined by HT $>225 \mathrm{GeV}$ and MET $>100 \mathrm{GeV}$.

In both regions, data is compared with the expected backgrounds and shows good agreement (see Fig. 4), making it possible to place limits on different models for new physics. Here, the analysis is interpreted in terms of cross-section limits in the MSSM, for which 4 mass spectra are chosen (see Table 3) with squark and gluino masses not yet ruled out by previous searches and with no mSUGRA solution. In Table 3, are listed for all 4 mass spectra, the set 95\% CL cross-section upper limits, and the limits to the Pythia leading order cross sections for inclusive production of squarks and gluinos, showing that spectra S2 and S3 are excluded, while S1 and S4 are not. 

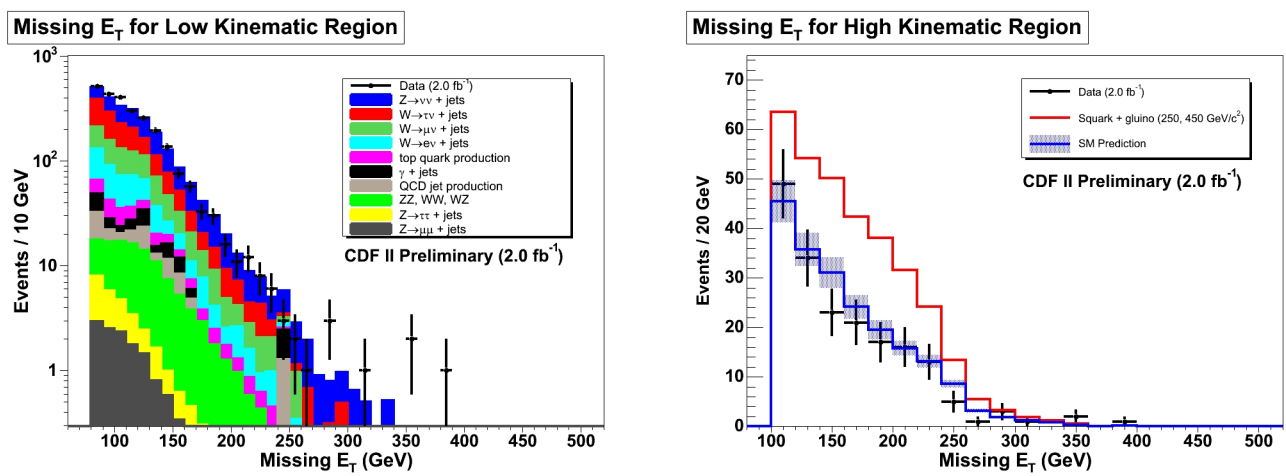

Figure 4: The left (right) plot shows the MET distribution of data and expected backgrounds for events in the low (high) kinematic regions defined by HT and MET requirements. The MET distribution of a signal corresponding to the $\mathrm{S} 2$ spectrum is also shown on the right plot.

\begin{tabular}{|c||c|c|c|c|c|c|}
\hline $\begin{array}{c}\text { SUSY } \\
\text { Spectrum }\end{array}$ & M(squark) & M(gluino) & $\begin{array}{c}\text { A priori limit } \\
(\mathrm{pb})\end{array}$ & $\begin{array}{c}\text { Observed limit } \\
(\mathrm{pb})\end{array}$ & $\begin{array}{c}\text { PYTHIA LO } \\
(\mathrm{pb})\end{array}$ & ratio \\
\hline S1 & 320 & 390 & 0.53 & 0.37 & 0.36 & 1.03 \\
\hline S2 & 250 & 450 & 0.90 & 0.62 & 1.73 & 0.36 \\
\hline S3 & 220 & 520 & 1.94 & 1.33 & 3.21 & 0.41 \\
\hline S4 & 120 & 550 & 78.9 & 73.8 & 57.4 & 1.29 \\
\hline
\end{tabular}

Table 3: CDF 95\% CL cross-section upper limite of inclusive $\tilde{q}+\tilde{g}$ production, compared to LO calculations

The leptoquark interpretation of this analysis has also been presented at this conference [5].

\section{Conclusions}

CDF and D0 have searched for squarks and gluinos in jets + MET final states on up to $2.1 \mathrm{fb}^{-1}$ data samples, leading to limits on the production of such particles. The combination of these limits is in progress. Although there is no evidence of SUSY as of yet, each experiment already has over $6 \mathrm{fb}^{-1}$ of recorded data and keeps taking high quality data. The search for SUSY continues.

\section{References}

[1] see for instance, P. Fayet et al., Phys. Rep. 32, 249 (1977); H.P. Nilles, Phys. Rep. 110, 1 (1984)

[2] V. Abazov et al., Search for squarks and gluinos in events with jets and missing transverse energy using $2.1 \mathrm{fb}^{-1}$ of ppbar collision data at sqrt(s)=1.96 TeV, Phys. Lett. B 660, 449 (2008)

[3] T. Aaltonen et al., Inclusive Search for Squark and Gluino Production in $p$ anti-p Collisions at $s^{* *}(1 / 2)=1.96-\mathrm{TeV}$, Phys. Rev. Lett. 102, 121801 (2009)

[4] V. Abazov et al., Search for squark production in events with jets, hadronically decaying tau leptons and missing transverse energy at sqrt $(s)=1.96 \mathrm{TeV}$, accepted for publication in Phys. Lett. B, [arXiv:0905.4086].

[5] Th. Nunnemann, Search for Leptoquark and Compositeness at the Tevatron, in these proceedings. 